\title{
Nitric oxide stimulates early egress of Toxoplasma gondii tachyzoites from human foreskin fibroblast cells
}

Xinlei Yan ${ }^{1,2}$, Yongsheng $\mathrm{Ji}^{1,2}$, Xianyong Liu ${ }^{1,2,3}$ and Xun Suo ${ }^{1,2,3^{*}}$

\begin{abstract}
Background: Egress is a vital step in the life cycle of Toxoplasma gondii which attracts attentions of many groups. Previous studies have shown that exogenous nitric oxide (NO) stimulates the early egress of $T$. gondii from infected peritoneal macrophages, a kind of immune cells. However, because Toxoplasma forms cysts in brain and muscle tissues, the development of autonomous immunity in non-immune cells is vital for limiting parasite burden and cyst formation. Therefore, we attempted to investigate whether exogenous NO could induce the early egress of $T$. gondii from infected non-immune cells.
\end{abstract}

Methods: T. gondii tachyzoites were cultured in human foreskin fibroblast (HFF) cells and were then treated with NO released by sodium nitroferricyanide (III) dihydrate (SNP). The egressed parasites were analysed by flow cytometry.

Results: The results showed that NO induced the early egress of parasites from HFF cells before completing their intracellular life cycles. We also found that the occurrence of egress was dependent on intracellular calcium $\left(\mathrm{Ca}^{2+}\right)$ levels and the mobility of the parasite. Compared with freshly isolated tachyzoites, the developmental ability and virulence of egressed tachyzoites presented no difference.

Conclusions: Taken together, our findings demonstrate a novel assay for the analysis of egress signalling mechanisms and an avenue of parasite clearance by hosts of T. gondii.

Keywords: Toxoplasma gondii, Nitric oxide, Non-immune cells

\section{Background}

Toxoplasma gondii is an obligate intracellular apicomplexan parasite that infects a wide range of vertebrate hosts including humans [1]. One third of the world's population has been reported to be chronically infected by Toxoplasma [2]. Immuno-compromised individuals, such as those with acquired immunodeficiency syndrome (AIDS), and transplant patients with acute or reactivated infections can develop severe infections, which may even lead to death [3]. A few of the devastating consequences caused by the parasite are due to lysis of the host cell during egress [4]. Egress of T. gondii was initially studied by inducing elevated levels of intracellular

\footnotetext{
* Correspondence: suoxun@cau.edu.cn

'State Key Laboratory of Agrobiotechnology, China Agricultural University, Beijing 100193, China

${ }^{2}$ National Animal Protozoa Laboratory \& College of Veterinary Medicine,

China Agricultural University, Beijing 100193, China

Full list of author information is available at the end of the article
}

calcium $\left(\mathrm{Ca}^{2+}\right)$ by ionophore A23187 [5]. Ethanol was also used to produce the secretion of microneme proteins, which results in the early egress of the parasite $[6,7]$. Another chemical, dithiothreitol (DTT), causes an acute egress of tachyzoites within $60 \mathrm{sec}$ by activating isoforms of the highly concentrated nucleoside triphosphate hydrolase (NTPase) [8]. In addition, a type of potassium ionophore triggers egress by causing an increase in the cytoplasmic $\mathrm{Ca}^{2+}$ levels within the parasite through the inositol-1,4,5-triphosphate $\left(\mathrm{IP}_{3}\right)$ pathway [9].

One of the characterised mechanisms of resistance to T. gondii in human non-immune cells involves a disruption of the intracellular life cycle of the parasite. Recently, many studies have focused on early egress from non-immune cells induced by immune molecules. Death receptor ligation in $T$. gondii infected cells results in the early egress of infectious parasites via an active process mediated by the release of intracellular $\mathrm{Ca}^{2+}[10]$. In addition, 
interferon- $\gamma$ (IFN- $\gamma$ )-induced cell death leads to early egress of Toxoplasma, which may promote the clearance of the parasite by immune cells [11].

Nitric oxide (NO) is produced by a number of different cells in response to cytokine stimulation and has been found to play roles in immunologically mediated protection against a growing list of protozoans, including T. gondii [12]. A previous study indicated that NO production during an acute infection with $T$. gondii can kill intracellular parasites [13], and the opposing effects of $\mathrm{NO}$ on the parasite contributed towards the establishment of a chronic state of host parasite equilibrium [14]. Moreover, when the parasites replicate in microlia, their multiplication could be prevented by activating the cells with IFN- $\gamma$ or lipopolysaccharide (LPS), which is a treatment that upregulates upregulate NO synthase activity [15].

Our recent study uncovered another effect of $\mathrm{NO}$ against $T$. gondii: exogenous NO induced the the early exit of tachyzoites from infected macrophages [16]. This finding introduced another type of immunemediated egress for Toxoplasma, which may become a new means for the study of parasite clearance by the immune system of host cells. However, the previous study did not determine whether NO could induce egress of tachyzoites from non-immune cells and exposed little information on the mechanism of this immune-mediated egress. In this study, we attempted to determine whether NO could induce the egress of $T$. gondii tachyzoites from non-immune cells and investigated the mechanism of NO-induced egress. Our results showed that NO could trigger the early egress of $T$. gondii tachyzoites from infected human foreskin fibroblast (HFF) cells by elevating the concentration of the cytoplasmic $\mathrm{Ca}^{2+}$ of the parasites and that the occurrence of egress required the parasite motility. Moreover, virulence of the egressed tachyzoites was not decreased. Taken together, our discovery presents a novel assay for the analysis of the signalling mechanisms of egress and the study of parasite clearance by hosts of T. gondii.

\section{Methods}

\section{Parasites and cell culture}

Two T. gondii strains were used: strain $\mathrm{RH}$ and a transgenic strain, RH-YFP, which stably expressed yellow fluorescent protein (YFP) [16]. The strains were grown as tachyzoites in monolayer cultures of HFF cells in Dulbecco's modified Eagle's medium (DMEM) supplemented with $10 \%$ foetal bovine serum (FBS). The cultures were maintained at $37{ }^{\circ} \mathrm{C}$ in a $5 \% \mathrm{CO}_{2}$ atmosphere. Parasite cultures were carried out in 25$\mathrm{cm}^{2}$ tissue culture flasks, and egress assays were conducted in 24-well tissue culture plates.

\section{Animals and ethical approval}

C57BL/6 mice (6-8-weeks old) were maintained in a pathogen-free facility. All animal research was approved by the Beijing Association for Science and Technology (approval ID SYXK (Beijing) 2007-0023) and complied with the guidelines of the Beijing Laboratory Animal Welfare and Ethics of the Beijing Administration Committee of Laboratory Animals.

\section{SNP-induced egress assay}

RH-YFP parasites $\left(2 \times 10^{5}\right)$ were allowed to infect HFF cells for $2 \mathrm{~h}$. Free parasites were washed with PBS three times. After $36 \mathrm{~h}$ of growth at $37{ }^{\circ} \mathrm{C}$, the infected HFF cells were then exposed to either different concentrations of sodium nitroferricyanide (III) dihydrate (Sigma) or DMEM carrier for various durations. After treatments, the numbers of free tachyzoites were counted by flow cytometry (C6, Accuri Cytometers, Inc.).

\section{Video microscopy}

The HFF cells were treated with $40 \mathrm{mM}$ SNP $36 \mathrm{~h}$ after infection with RH-YFP strain tachyzoites $\left(2 \times 10^{5}\right)$. The progress of egress was observed under a light microscope and video was recorded.

\section{Inhibition assays}

For inhibition assays, two calcium inhibitors, BAPTA, an extracellular $\mathrm{Ca}^{2+}$ chelator and BAPTA-AM, an intracellular $\mathrm{Ca}^{2+}$ chelator, were utilised. HFF cells were infected with RH-YFP parasites $\left(2 \times 10^{5}\right)$ for $36 \mathrm{~h}$. Cell cultures were then pre-incubated with $10 \mu \mathrm{M}$ BAPTA or BAPTAAM for $30 \mathrm{~min}$. After the pre-incubation, $40 \mathrm{mM}$ SNP was added to the cultures for $30 \mathrm{~min}$, and the numbers of egressed parasites were determined by flow cytometry. To test the effect of disrupting actin-dependent parasite motility on NO-induced egress, intracellular tachyzoites were treated for $30 \mathrm{~min}$ with $10 \mu \mathrm{M}$ cytochalasin D (Sigma), then co-cultured with $40 \mathrm{mM}$ SNP for $30 \mathrm{~min}$. The efficiency of egress was then determined as described above.

\section{Virulence assay}

RH-YFP tachyzoites were allowed to grow in HFF cells for $36 \mathrm{~h}$ in $25-\mathrm{cm}^{2}$ tissue culture flasks and were then treated with $40 \mathrm{mM}$ SNP for $30 \mathrm{~min}$. The cell culture medium containing the egressed parasites was centrifuged at $1500 \mathrm{rpm}$ for $10 \mathrm{~min}$. The number of tachyzoites was counted using a blood cell counting plate. For in vitro assays, $2 \times 10^{5}$ egressed tachyzoites were added to HFF cells for $24 \mathrm{~h}$, and the number of parasites per vacuole was determined by microscopic examination [17]. For in vivo assay, the egressed tachyzoites were used to infect C57BL/ 6 mice intraperitoneally, and the survival rate after acute challenge was confirmed. 


\section{Statistical analysis}

All statistical analyses were processed by the SPSS15.0 Data Editor software (SPSS Inc., Chicago, IL), and all data are expressed as means \pm SD values.

\section{Results}

SNP-released NO triggered early egress of $T$. gondii tachyzoites from infected HFF cells

A previous study reported that exogenous NO released by SNP could trigger the egress of $T$. gondii tachyzoites from infected peritoneal macrophages [16]. To investigate whether NO could induce egress of tachyzoite from infected-HFF cells, we treated T. gondii tachyzoite-infected HFFs with different concentrations of SNP for different durations as described in the literature [16]. As shown in Fig. 1a, infected HFF cells incubated with SNP displayed increased parasite egress compared with the control group. When infected HFF cells were incubated with $40 \mathrm{mM}$ SNP for up to $30 \mathrm{~min}$, a maximum number of free parasites was observed. By virtue of the video record from microscopy, we observed a large number of tachyzoites egressed from infected cells during treatment with $40 \mathrm{mM} \mathrm{SNP}$ (Fig. 1b).

\section{NO-induced egress was dependent on calcium signalling of the parasites}

Calcium released from intracellular pools of the parasites was necessary and sufficient to induce many critical events for egress, as was confirmed by many studies [18]. To test whether an increase of parasite $\mathrm{Ca}^{2+}$ levels was required during $\mathrm{NO}$-induced egress, the membrane- permeable calcium chelator BAPTA-AM was co-cultured with infected cells before SNP treatment. It was found that treatment of the parasites with BAPTA-AM completely prevented egress (Fig. 2a). Interestingly, we found that the BAPTA chelation of the extracellular $\mathrm{Ca}^{2+}$ of the parasite had no effects on the NO-induced egress (Fig. 2b), which suggested that rather than an increase in the $\mathrm{Ca}^{2+}$ flux in the host cell cytoplasm, an increase in the parasite $\mathrm{Ca}^{2+}$ levels was critical for Toxoplasma egress.

\section{Parasite motility was essential for NO-induced egress}

Gliding motility is essential for $T$. gondii tachyzoites to traverse across substrates, migrate through tissues, and invade into and finally egress from their host cells [4]. Thus, we hypothesised that the effect of NO on egress should be dependent on the motility machinery of the parasite. To test this hypothesis, we studied the effect of the actin inhibitor cytochalasin D, which can completely block the actin microfilaments and gliding motility of $T$. gondii [19]. When intracellular tachyzoites were treated with NO in the presence of cytochalasin D, the parasites lost their ability to egress from HFF cells (Figs. 3a, b, and c). This result suggested that gliding motility is a crucial step in NO-induced egress.

\section{Occurrence of NO-induced egress had no effects on viru- lence of egressed parasites}

Previous studies on the egress of $T$. gondii reported that death receptor ligation could induce parasite egress. However, the egressed tachyzoites would then rapidly infect

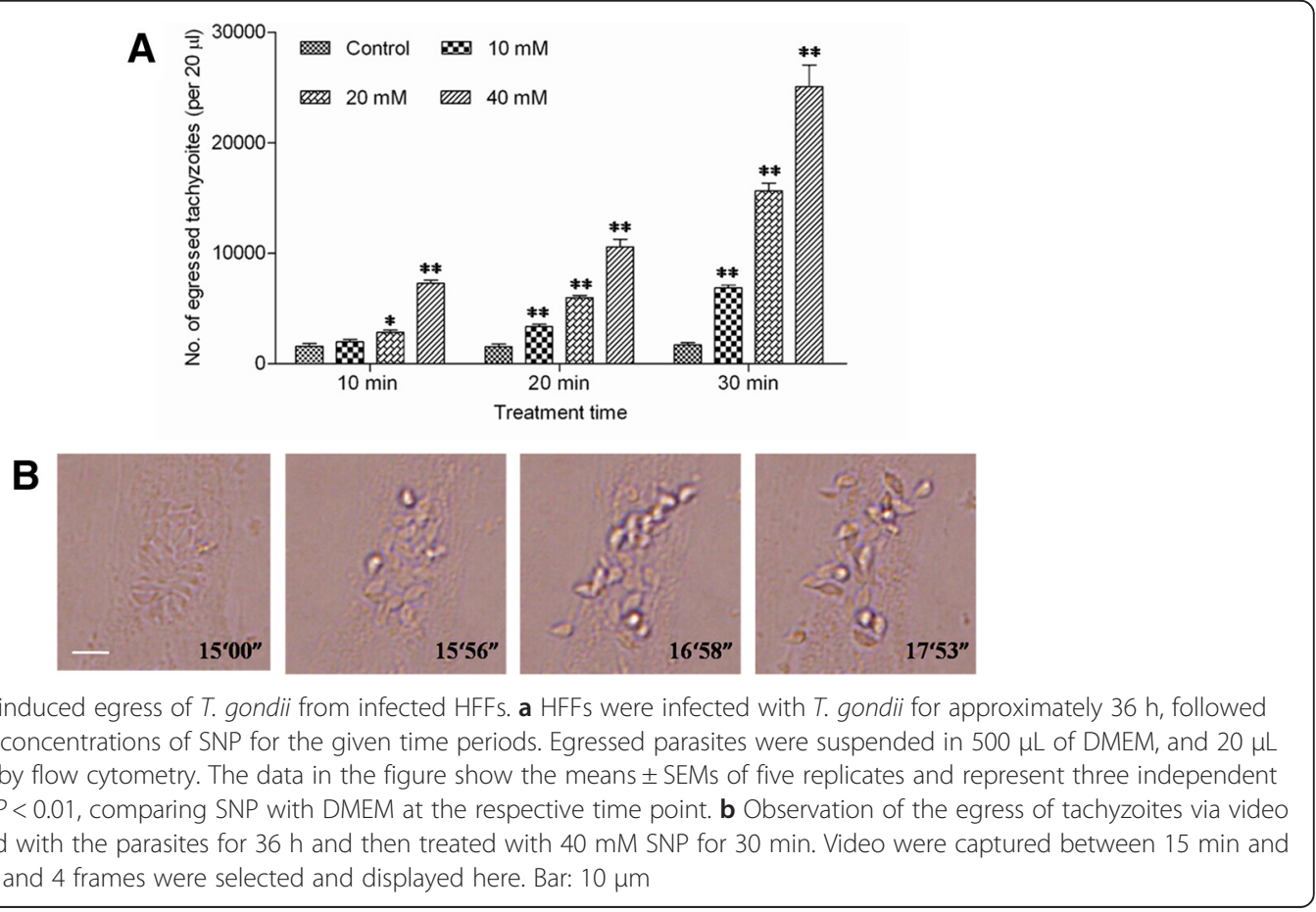




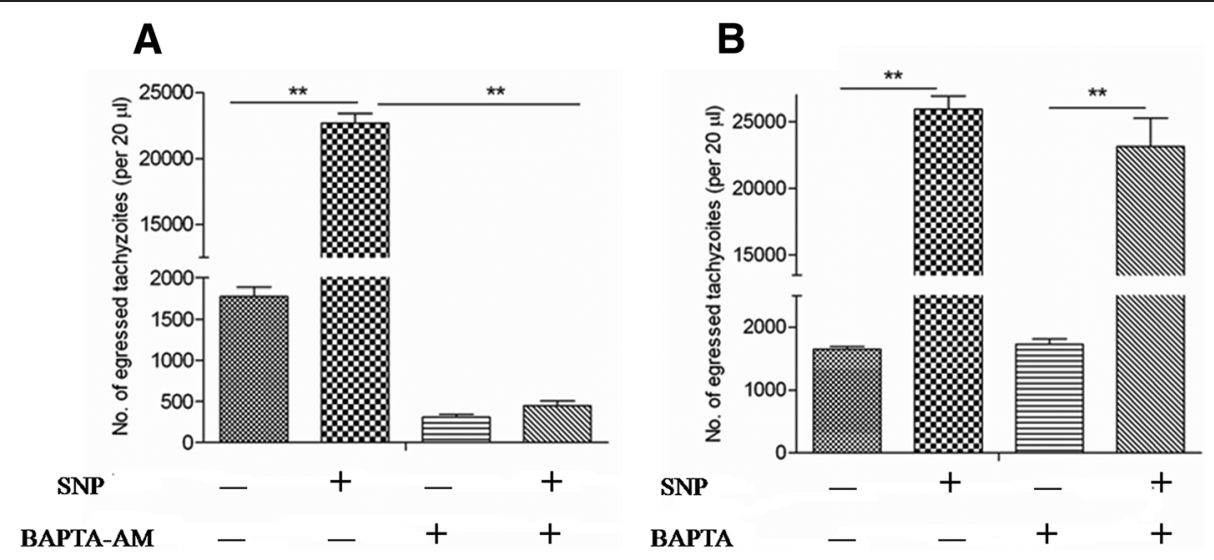

Fig. 2 Exogenous NO-induced egress of T. gondii from HFFs was dependent on calcium flux of the parasite. Tachyzoite-infected HFFs were pre-treated with BAPTA-AM (a) or BAPTA (b) to chelate calcium in the parasite cytosol or host cells, respectively, before NO-induced egress assay as described in the Methods section. Egressed parasites were suspended with $500 \mu \mathrm{L}$ of DMEM, and $20 \mu \mathrm{L}$ suspensions were analysed by flow cytometry. The data in the figure show the means \pm SEMs of four replicates and represent three independent experiments, ${ }^{* *} P<0.01$

surrounding cells, including Ag-specific effector cells, which could potentially contribute to a local dissemination of the infection [10]. To investigate whether the parasites were still infectious after NO-induced egress, we collected egressed tachyzoites to infect newly prepared HFF cells or to intraperitoneally inoculate $\mathrm{C} 57 \mathrm{BL} / 6$ mice. It was found that both the developmental ability in host cells (Fig. 4a) and the lethal infection in laboratory mice (Fig. 4b) by parasites produced via NO-induced egress were not different from those of normal parasites.

\section{Discussion}

Our results demonstrated that NO released by SNP was able to trigger early egress of $T$. gondii tachyzoites from non-immune cells in vitro. Moreover, these processes were revealed to be $\mathrm{Ca}^{2+}$-dependent as inhibition of parasite intracellular $\mathrm{Ca}^{2+}$ prevented this phenomenon. Our further experiments showed that the occurrence of egress required parasite motility, and the egressed tachyzoites lost no virulence. Taken together, our study investigated the mechanism of NO-induced tachyzoite egress and presented a new mechanism of parasite clearance.

T. gondii establishes a life-long infection in hosts by forming cysts in brain and muscle tissues. Therefore, cell-autonomous immunity in non-immune cells is crucial for limiting parasite burden and cyst formation [11]. Previous studies have demonstrated that death receptor ligation and IFN- $\gamma$ induce the egress of $T$. gondii tachyzoites from
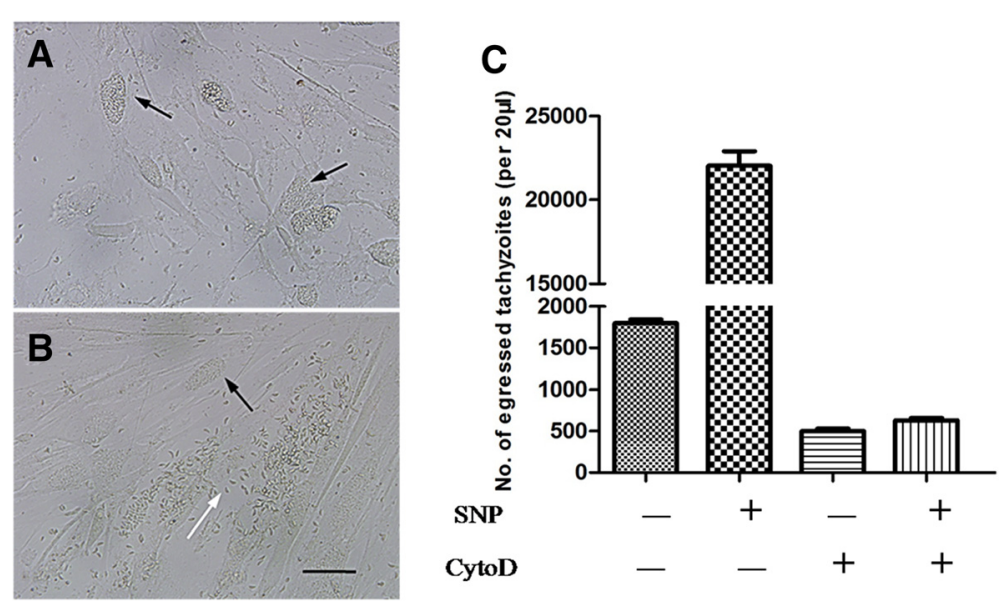

Fig. 3 Impairment of parasitic motility impeded NO-induced egress of T. gondii from HFFs. NO-induced egress after pre-treatment with cytochalasin D (a) or DMEM (b) was observed by microscopy. Intact parasitophorous vacuole (black arrow), lytic parasitophorous vacuole (white arrow). Bar: $20 \mu \mathrm{m}$. c Tachyzoite- infected HFFs were pre-treated with cytochalasin D before NO-induced egress assay. Egressed parasites were suspended with $500 \mu \mathrm{L}$ of DMEM, and $20 \mu \mathrm{L}$ suspensions were analysed by flow cytometry. The data in the figure show the means \pm SEMs of five replicates and represent three independent experiments, ${ }^{* *} P<0.01$ 

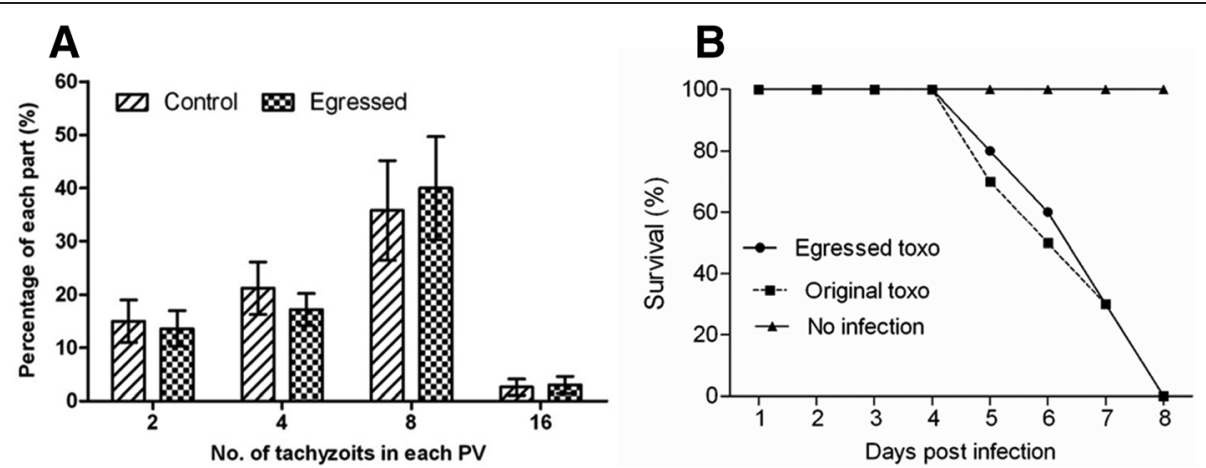

Fig. 4 Egressed parasites had similar in vitro growth and virulence as control parasites. a HFFs were infected with egressed T. gondii induced by NO or naturally released parasites. Twenty-four hours later, the cells were fixed and the number of parasites per vacuole in eight fields was counted and expressed as a percentage of the total number of parasites in the field. The data in the figure show the means \pm SDs of five replicates and represent three independent experiments. b C57BL/6 mice were i.p infected with $10^{3}$ parasites and the survival rate was recorded every day

non-immune cells in vitro $[10,11]$. Here, we found that $\mathrm{NO}$ is another immune molecule that can induce the early egress of $T$. gondii tachyzoites from non-immune cells. Moreover, we found that the the egressed tachyzoites lost no virulence, but $\mathrm{NO}$ stimulated the egress of parasites from HFF cells $36 \mathrm{~h}$ after infection, long before natural egress occurred, and thereby disturbed the proliferation of the parasite. If this kind of immune-mediated egress existed in vivo, it may limit replication of the tachyzoites in non-immune cells and lower the parasite burden of the host. Moreover, the occurrence of egress before complete proliferation shortens the time of the parasite in parasitophorous vacuole which promotes the clearance of the parasites by immune cells of the hosts, like macrophages.

The study of the egress of $T$. gondii has attracted attention from many research groups for nearly four decades. T. gondii egress is a crucial step in the life cycle of the parasite because it allows parasites to shuttle into neighbouring cells for further development. Many studies have shown that intracellular $\mathrm{Ca}^{2+}$ levels of the parasite play important roles in regulating the egress of parasites from host cells in response to a variety of extracellular signals [20-22]. Our study found that rather than an increase in the $\mathrm{Ca}^{2+}$ levels in the host cell, an increase of $\mathrm{Ca}^{2+}$ levels in the parasite was critical for the NO-induced egress of $T$. gondii tachyzoites. Moreover, in Plasmodium falciparum, an increase in the intracellular $\mathrm{Ca}^{2+}$ levels was found to stimulate parasite egress from infected erythrocytes [23]. Another study showed that the addition of $\mathrm{Ca}^{2+}$ ionophores induces the rapid egress of Neospora caninum tachyzoites from bovine endothelial cells [24]. Recent research on Eimeria tenella revealed that ethanol, a reagent that is able to elevate intracellular $\mathrm{Ca}^{2+}$ levels, induces sporozoite egress from primary chicken kidney cells [25]. Consistent with the findings on $T$. gondii, we hypothesised that $\mathrm{Ca}^{2+}$ is a universal signalling molecule of the egress process in apicomplexan parasites.

\section{Conclusions}

In conclusion, our research demonstrated that NO is an immune molecule that could induce the early egress of T. gondii tachyzoites from non-immune cells and investigated the mechanism of this immune-mediated egress. Future studies will address whether these immune molecules could induce parasite egress from feline intestinal epithelial cells in vitro and whether the induction of early parasite egress from non-immune cells by immune molecules could occur in vivo.

\section{Competing interests}

The authors declare that they have no competing interests.

\section{Authors' contributions}

$X L Y$ and XS designed this study and analysed the data; XLY carried out the experiments, performed the statistical analysis and drafted the manuscript; YSJ contributed to help perform the statistical analysis and help to draft the manuscript; XYL helped to design the study and helped to draft the manuscript; XS supervised the study implementation and revised the manuscript. All authors read and approved the final version of the manuscript.

\section{Acknowledgments}

This research was supported by the National Natural Science Foundation of China (Project number 31172309).

\section{Author details}

${ }^{1}$ State Key Laboratory of Agrobiotechnology, China Agricultural University, Beijing 100193, China. ${ }^{2}$ National Animal Protozoa Laboratory \& College of Veterinary Medicine, China Agricultural University, Beijing 100193, China. ${ }^{3}$ Key Laboratory of Zoonosis of Ministry of Agriculture \& College of Veterinary Medicine, China Agricultural University, Beijing 100193, China.

Received: 13 June 2015 Accepted: 6 August 2015

Published online: 13 August 2015

\section{References}

1. Black MW, Boothroyd JC. Lytic cycle of Toxoplasma gondii. Microbiol Mol Biol Rev. 2000;64:607-23.

2. Okada T, Marmansari D, Li ZM, Adilbish A, Canko S, Ueno A, et al. A novel dense granule protein, GRA22, is involved in regulating parasite egress in Toxoplasma gondii. Mol Biochem Parasit. 2013;189:5-13.

3. Luft BJ, Remington JS. Toxoplasmic Encephalitis in Aids. Clin Infect Dis. 1992;15:211-22.

4. Sibley LD. How apicomplexan parasites move in and out of cells. Curr Opin Biotech. 2010;21:592-8. 
5. Endo T, Sethi KK, Piekarski G. Toxoplasma gondii: calcium ionophore A23187-mediated exit of trophozoites from infected murine macrophages. Exp Parasitol. 1982;53:179-88.

6. Carruthers VB, Moreno SN, Sibley LD. Ethanol and acetaldehyde elevate intracellular $\left[\mathrm{Ca}^{2+}\right]$ and stimulate microneme discharge in Toxoplasma gondii. Biochem J. 1999;342:379-86.

7. Kafsack BF, Pena JD, Coppens I, Ravindran S, Boothroyd JC, Carruthers VB Rapid membrane disruption by a perforin-like protein facilitates parasite exit from host cells. Science. 2009;323:530-3.

8. Stommel EW, Ely KH, Schwartzman JD, Kasper LH. Toxoplasma gondii: Dithiol-induced $\mathrm{Ca}^{2+}$ flux causes egress of parasites from the parasitophorous vacuole. Exp Parasitol. 1997;87:88-97.

9. Fruth IA, Arrizabalaga G. Toxoplasma gondii: Induction of egress by the potassium ionophore nigericin. Int J Parasitol. 2007;37:1559-67.

10. Persson EK, Agnarson AM, Lambert H, Hitziger N, Yagita H, Chambers BJ, et al. Death receptor ligation or exposure to perforin trigger rapid egress of the intracellular parasite Toxoplasma gondii. J Immunol. 2007:179:8357-65.

11. Niedelman W, Sprokholt JK, Clough B, Frickel EM, Saeij JP. Cell death of gamma interferon-stimulated human fibroblasts upon Toxoplasma gondii infection induces early parasite egress and limits parasite replication. Infect Immun. 2013;81:4341-9.

12. James SL. Role of Nitric-Oxide in Parasitic Infections. Microbiol Rev 1995;59:533.

13. Khan IA, Schwartzman JD, Matsuura T, Kasper LH. A dichotomous role for nitric oxide during acute Toxoplasma gondii infection in mice. P National Acad Sci USA. 1997;94:13955-60.

14. Hayashi S, Chan CC, Gazzinelli R, Roberge FG. Contribution of nitric oxide to the host parasite equilibrium in toxoplasmosis. J Immunol. 1996;156:1476-81.

15. Chao CC, Anderson WR, Hu SX, Gekker G, Martella A, Peterson PK. Activated Microglia Inhibit Multiplication of Toxoplasma gondii Via a Nitric-Oxide Mechanism. Clin Immunol Immunopathol. 1993;67:178-83.

16. Ji YS, Sun XM, Liu XY, Suo X. Toxoplasma gondii: effects of exogenous nitric oxide on egress of tachyzoites from infected macrophages. Exp Parasitol. 2013;133:70-4

17. Fux B, Nawas J, Khan A, Gill DB, Su CL, Sibley LD. Toxoplasma gondii strains defective in oral transmission are also defective in developmental stage differentiation. Infect Immun. 2007:75:2580-90.

18. Arrizabalaga G, Boothroyd JC. Role of calcium during Toxoplasma gondii invasion and egress. Int J Parasitol. 2004;34:361-8.

19. Dobrowolski JM, Sibley LD. Toxoplasma invasion of mammalian cells is powered by the actin cytoskeleton of the parasite. Cell. 1996;84:933-9.

20. Black MW, Arrizabalaga G, Boothroyd JC. lonophore-resistant mutants of Toxoplasma gondii reveal host cell permeabilization as an early event in egress. Molr Cellr Biol. 2000;20:9399-408.

21. Caldas LA, de Souza W, Attias M. Calcium ionophore-induced egress of Toxoplasma gondii shortly after host cell invasion. Vet Parasitol. 2007;147:210-20.

22. Nagamune K, Hicks LM, Fux B, Brossier F, Chini EN, Sibley LD. Abscisic acid controls calcium-dependent egress and development in Toxoplasma gondii. Nature. 2008:451:207-10

23. Glushakova S, Lizunov V, Blank PS, Melikov K, Humphrey G, Zimmerberg J. Cytoplasmic free $\mathrm{Ca}^{2+}$ is essential for multiple steps in malaria parasite egress from infected erythrocytes. Malaria J. 2013;12:41.

24. Behrendt JH, Taubert A, Zahner H, Hermosilla C. Studies on synchronous egress of coccidian parasites (Neospora caninum, Toxoplasma gondii, Eimeria bovis) from bovine endothelial host cells mediated by calcium ionophore A23187. Vet Res Commun. 2008:32:325-32

25. Yan X, Liu X, Ji Y, Tao G, Suo X. Ethanol and isopropanol trigger rapid egress of intracellular Eimeria tenella sporozoites. Parasitol Res. 2015;114:625-30.

\section{Submit your next manuscript to BioMed Central and take full advantage of:}

- Convenient online submission

- Thorough peer review

- No space constraints or color figure charges

- Immediate publication on acceptance

- Inclusion in PubMed, CAS, Scopus and Google Scholar

- Research which is freely available for redistribution

Submit your manuscript at www.biomedcentral.com/submit 